\title{
La concentración del poder económico en los Estados Unidos y sus proyec- ciones en América latina
}

CELs O F UR TA D o es Profesor Asociado de la Facultad de Leyes y Ciencias Económicas de la Universidad de Paris y Profesor Asociado del Instituto de Estudios Internacionales de la Universidad de Chile. Sirvió como Ministro de Planificación en el Gobierno del Presiclente Joao Goulart; anteriormente fuc Director del Banco de Desarrollo del Brasil y de sudene. Sus principales publicaciones incluyen, Formaçao economica do brasil, 1959; Dialectica del Desarrollo, 1965; Teoria e politica do desenvolvimento economico, 1967.

Las complejas relaciones económicas que existen entre Estados Unidos y los países latinoamericanos son, evidentemente, el resultado de una larga evolución histórica. La diversidad de las estructuras socioeconómicas surgidas en las dos regiones en el periodo colonial y' en la primera fase de la Revolución Industrial, así como la proximidad geográfica, responden en buena medida al carácter de dependencia que marca tales relaciones en forma cacla rez más nítida en el siglo actual. En el presente artículo se admite ese estado de dependencia como evidente, lo que no significa que las peculiaridades que presenta en cada subregión hayan sido adecuadamente estudiadas, y aun menos que se haya analizado la significación exacta de esa dependencia desde el punto de vista del desarrollo o estancamiento de los distintos países. Tampoco consideraremos las opciones concretas que se presentan a éste o aquel país, vis-à-vis los aspectos negativos de la dependencia ${ }^{1}$. El estudio de casos particulares exige una movilización de informaciones y una amplitud de análisis que por mucho superan el objetivo del presente trabajo, que es contribuir a una mejor percepción del marco general del proceso de dependencia. En efecto, cl objetivo del presente artículo es limitädo; se pretende contestar a esta pregunta: ¿hasta qué punto y en qué forma, las modificaciones estruc-

¿Véase Osvaldo Sunkel. 'Politica Nacional de Desarrollo y Dependencia Externa', en Estudios Intcrnacionales, Año 1, No 1; : Colso Iiurlaclo, Subdesarrollo y Estacamiento en América latina, capitulo It (EUDkB, 196il). 
turales recientes en la economía de los Estados Unidos, resultàntes de la rápida concentración del poder económico, estän influyendo en el proceso histórico latinoamericano?

LAS FORMAS TRADICIONALES DE LA CONCENTRACIÓN DEL PÓEER ECONÓMICO

La estructura de la economía norteamericana, en particular de su sector manufacturero, muestra un elevado grado de concentración. Iniciado en los últimos decenios del sigło pasado, cuando el mércado nacional ya había alcanzado un alto grado de integración, el proceso de concentración se presentó bajo un doble aspecto de control progresivo -por una empresa de un mercado determinado- de un producto o de una clase đe productos y de articulación bajo un solo grupo económico de las distintas fases de un proceso productivo, desde la obtención de una materia prima hasta el suministro de un producto final a su consumidor. Estas son las modalidades clásicas de integración horizontal y vertical, que forman la base de la teoria de las llamadas formas imperfectas del mercado.

Una importante legislación surgío en los Estados Unidos, a partir de fines del siglo pasado ${ }^{2}$, con el objeto de evitar que las formas de poder, que surgian de las nuevas estructuras de mercado, fueran utilizadas contra el interés público, y aun cuando prevalece en los Estados Unidos, tanto en el ambiente universitario como en la Corte Suprema, una actitud doctrinaria en contra de la concentración del poder económico, esta tendencia ha seguido acentuándose en los últimos decenios. Esto no significa necesariamente que la eliminación de los competidores en cada mercado haya proseguido como en el comienzo del siglo. En realidad, las formas clásicas del poder monopolistico han pasado a un segundo plano; tiende ahora a prevalecer el esquema de organización oligopolística de los mércádos, que es compatible con la política de precios administrados que conviene a las grandes empresas y permite la unión de varios grupos en un esfuerzo conjunto para condicionar el comportamiento del consumidor sin conflicto con la legislación vigente ${ }^{3}$. Además, han aparecido otras formas de concentración, que escapan a los esquemas tradicionales de control judicial, a las cuales nos referiremós más adelante.

Cualquiera que sea el criterio adoptado para medir el fenómeno, siempre se llega a la conclusión de que un número reducido de socie-

'El Sherman Act fue aprobado en 1890.

sPara un análisis en que se identifican los 35 oligopolius más significativos de la industria manufacturera de Estados Unidos, véase William G. Shepherd. 'Trends of Concentration in American Manufacturing Industries, 1947-58' en Review of Economics and Statistics, mago 1964 . 
dades anónimas tiende a controlar una fracción creciente de poder de decisión en la economía de los Estados Unidos. El mejor criterio para medir el fenómeno de la concentración es el monto relativo de los activos netos de capital de làs empresas, aunque se obtienen resultados muy similares si se adopta como,base de cálculo el monto relativo de las ventast. Adoptado el primer criterio se comprueba que las 100 mayores firmas americanas ocupan una posición de predominio creciente en la economía americana. Esa tendencia parece haberse acentuado en la postguerra. En efecto, entre 1929 y 1947, la participación de las mayores 100 firmas en el control de los activos netos de capital de las manufacturas habría pasado tan sólo de 44 a 46 por ciento, en tanto que entre 1947 y 1962, el incremento ha sido de 46 a 57 por ciento.

El grado de concentración del poder económico logrado en los Estados Unidos se desprende del hecho de que en 1962 los activos de las. 20 mayores sociedades anónimas manufactureras eran tan grandes como los de las 419.000 empresas más pequeñas, en un total de 420.000 empresas. Una cuarta parte de los activos netos pertenece a los 20 mayorés; la mitad a las 1.000 mayores y una cuarta parte a las 419.000 restantes. Otro indicador del mismo fenómeno es el hecho de que en 1963 las tres mayores empresas manufactureras obtuvieron ingresos brutos por un monto de $63 \mathrm{mil}$ millones de dólares, tánto como los ingresos brutos obtenidos por todos los agricultores del país ${ }^{0}$.

Ese proceso de concentración, que parece haberse acentuado ${ }^{7}$, debe ser estudiado con atención si pretendemos conocer los factores que determinan las transformaciones de la estructurá económica americana y captar la naturaleza de las relaciones entre ella y las economías latinoamericanas.

Entre los factores que actúan en el sentido de la concentración tienen papel relevante el tamaño creciente de las plantas (economfas

'Se obtienc resultado distinto en base a la mano de obra empleada, pucs el cocficiente de capital por pérsona empleada generalmente es mayor en las empresas grandes.

Véasc el abundante material cstadístico presentado por testigos ante el Subcomité sobre Antitrust y Monopolio del Senado de los Estados Unidos, particularmente las sesiones de julio a septicmbre de 1964. Los datos estadisticos incluidos en el presente artículo, cunndo no se indica explicitamente la fuente, han sido recogidos de esos testigos, particularmente los del Prof. Gardner Means, del Dr. John M. Blair, Economista Jefe del referido Subcomité, del Prof. Corwin D. Edwards. del Dr. Irvin Stelzer y del Prof. Ralph L. Nelson.

aLa General Mlofors, la mayor empresa del mundo, emplea 735.000 personas, tiene 1,3 millones de aecionistas en más de 80 paises $y$ opera plantas en 24 paises. Sus utilidades netas (después de pagados los impuestos) alcanzaron, en 1965, a 2,1 millones de dólares, siendo superior a los ingresos fiscales de 48 de los Estados de la Unión. Las ventas de la General Motors alcanzaron a 21 mil millones de dólares, nproximadamente igual a una tercera parte del ingreso nacional de todos los paises latinonnericanos juntos. Véasc Richard y Barber "The New Partnership, Big Governatent and Big Business, The New Republic, 13 de agosto de 1966.

TEn 1965 han sido absorbidas cerca de 1.000 empresas y en 1966 un número aún superior de absorciones se tenia coino probable. Richard y Barber, cit. 
de escala), la dimensión de los mercados, la tasa de crecimiento de esos mercados y el número medio de plantas que las empresas deciden operar.

Analizando la actual estructura de la economía americana se comprueba que el grado de concentración varía mucho de un mercado a otro. Para medir la concentración es corriente utilizar como base la participación de las cuatro mayores empresas en cada mercado. Si se define como mercado una de las mil y tantas clases de productos principales que salen de la industria manufacturera, el coeficiente de concentración varia, de 3 por ciento en la industria de bloques de concreto y ladrillos al 99 por ciento en el aluminio primario. El hecho más significativo que se desprende de esto es que no existe correlación positiva entre el grado de concentración y el aprovechamiento de las economías de escala de producción por las empresas dominantes, es decir, las cuatro mayores operando en los principales mercados. Al parecer, la operación de plantas que maximizan la eficiencia desde el punto de vista tecnológico, no exige un elevado grado de concentración, excepción hecha de las ramas industriales de importancia secundaria.

La tendencia a multiplicar el número de plantas similares, que se observa entre las firmas más poderosas, parece ser el principal factor responsable de la concentración. En efecto, las cuatro empresas dominantes, en las ramas más significativas, operan un promedio de más de 5 plantas con esquemas de producción similar, mientras las empresas menores operan un promedio apenas superior a una planta. Además, se observa que es en las industrias de mayor grado de concentración que la tendencia a operar un gran número de plantas similares se presenta con más énfasis. En efecto, en éstas (de 70 al 400 por ciento del mercado controlado por cuatro firmas), las dos terceras partes de las plantas "excedentarias" 8 pertenecen a las 4 firmas dominantes, mientras que en las industrias de concentración débil (30 por ciento o menos del mercado controlados por las 4 firmas mayores) menos de una quinta parte de las plantas "excedentarias" son operadas por las 4 empresas mayores.

Adlemás de operar un número elevado de plantas similares, las empresas dominantes también operan plantas de tamaño medio relativamente grande. En promedio, esas plantas son dos veces mayores que el promedio de las plantas de las demás empresas. Es interesante señalar que esta tendencia a operar plantas relativamente grandes no es una característica de las empresas dominantes de aquellas ramas

'Se define para fincs del análisis como planta 'excedentaria' toda aquella que duplica una ya existente cu la empresa. 
de más elevado coeficiente de concentración. Hay evidencia estaclística que apunta exactamente hacia la conclusión contraria: es en las industrias de menor concentración que se comprueba la tendencia a aumentar el tamaño medio de las plantas. En otras palabras: el ańtlisis de la estructura de la manufactura estadounidense parece indicar que el proceso de concentración actual se funda de manera secundaria en las economías de escala de producción.

Si se considera el proceso de concentración en el tiempo se constata que en mercados de más lento crecimiento es mayor el grado de concentración. También se observa una correlación negativa entre el tamaño relativo del mercado y el grado de concentración. Los dos fenómenos parecen estar relacionados, pues el mercado que crece lentamente tiende a perder importancia relativa en el conjunto de la economía. La concentración es más la resultante del lento crecimiento que del tamaño relativo original del mercado.

\section{El fenómeno de la Conglomeración}

En la medida en que avanzan los estudios de base empírica del proceso de concentración, se hace evidente que las teorias convencionales de las formas del mercado son de utilidad limitada para explicar el funcionamiento de una economía capitalista de elevado grado de desarrollo. El propósito directo de eliminar los competidores para obtener ganancias de monopolista, sólo se manifiesta en casos especiales. Por otro lado, tampoco existe base para afinmar que el imperativo tecnológico, fundamento de las economías de escala, es el factor determinante de la evolución estructural del sistema económico. Lo mismo se puede decir en relación a las economías que acompañan la integra. ción vertical: reducción de existencias, sincronización de fases productivas complementarias, etc. En otras palabras: los factores tecnológicos y económicos del proceso de integración que han sido identificados a partir de la teoría de la empresa, no van al fondo del problema. Sin que haya abandonado los canales tradicionales de la integración horizontal o vertical, el fenómeno de la concentración se realiza hoy día principalmente por la vía de la diversificación o conglomeración ${ }^{\theta}$.

Analizando la estructura de las grandes empresas se verifica de inmediato que ellas actúan en una multiplicidad de sectores, sin que exista necesariamente una relación de tipo tecnológico o económico

'Hoy día más del 70 por ciento de las fusiones son del tipo conglomerado, reuniendo en una sola empresa firmas que operan en ramas sin cualquiera relación mutua. Las consolidaciones entre competidores - tipo horizontal- suman tan sólo 12 por ciento de las fusiones, habiendo representado más del 30 por ciento al comienzo de los años 50". Richard y Barber, cit. 
entre los mismos. Más aún: el crecimiento reciente de las grandes empresas aumenta esa aparente desarticulación.

Si consideramos las 100 mayores sociedades anónimas que controlan más de la mitad de los activos netos de las manufacturas americanas, constatamos que ellas aparecen entre lis 4 mayores firmas de por lo menos la mitad de todos los mercados importantes. Por ejemplo: existen 66 clases de productos textiles considerados como mercados distintos; en más de la mitạd de esos mercados aparece entre las mayores 4 firmas por lo menos $l$ de las 100 mayores sociedades, anónimas del país. Existen 81 clases de productos qufmicos: en el 90 por ciento de ellas 1 de los 4 mayores productores pertenece a la familia de los 100 grandes, y asi sucesivamente.

En verdad, la ampliación del campo de actuación de las grandes empresas parecería ser la característica predominante de la presente evolución estructural de la economía americana. Comparando las 1.000 mayores finmas manufactureras que operaban en 1950, con las 1.000 mayores de 1962, se hace evidente la tendencia a la diversificación. En efecto, las firmas que producian tan sólo l clase de productos disminuyeron de 78 a 49 ; las que producian de 2 a 5 productos, descendieron de 354 a 223; por otro lado, las que producían de 16 a 50 productos aumentaron de 128 a 236 y las que producian más de 50 productos, pasaron de 8 a 15. De las 1.000 mayores firmas de 1950, las dos terceras partes estaban igualmente entre las 1.000 mayores de 1962. Si se las estudia individualmente, compruébase la misma tendencia a la ampliación del campo de actividad. Además, son las principales dentro de las 1.000 mayores firmas aquellas en que es más acentuada la tendencia a la diversificación.

La emergencia y rápida expansión de los conglomerados económi$\cos$-firmas que controlan múltiples actividades productivas no relacionadas- es el elemento dominante del actual proceso de concentración en los Estados Unidos. El método corriente de crecimiento de los conglomerados es la absorción de otras firmas en operación. Un buen ejemplo es la Textron, empresa originalmente textil que adquirio, en los últimos dos decenios, 70 otras empresas que operaban en 36 ramás de industrias diversas. Ese gran conglomerado-actúa hoy en una multiplicidad de industrias no relacionadas, comprendiendo desde la fabricación de helicópteros (mayor productor del país en este sector) hasta la crianza de gallinas, la fabricación de equipos para la industria óptica y la producción de aceites vegetales. Hary empresas que actúan simultáneamente en la metalurgia, el cine y el alquiler de coches, o que actúan en la minería y la radiodifusión. 
Desde el punto de vista de la teoría económica convencional, el conglomerado podría parecer una aberración, pues no se apoya en las economias de escala ni en las consabidas ventajas de la integración vertical. Pero su vitalidad es evidente y ya constituye la forma predominante de organización de la producción. Se basa él en dos principios. El primero es el mismo que ha dado origen a los fondos de financiación: invertir en múltiples sectores con un mínimo de interrelación es reducir el coeficiente de riesgo. El segundo principio indica que para luchar en un mercado particular, es más relevante el poder financiero que la participación relativa en ese mercado,

El conglomerado surge; así, como un fenómeno del capitalismo postcíclico. Eliminados los grandes ciclos de prosperidad y depresión, una inversión que se distribuya al azar entre un gran número de sectores productivos, se pone al abrigo de riesgos mayores. Siempre que se sostenga la demanda efectiva, las pérdidas ocasionales de ciertos sectores encontrarían compensación en las ganancias de otros. Por otro lado, una empresa de gran poder financiero, es invulnerable a presiones ejercidas en mercados en que ella ha aplicado una fracción pequeña de sus recursos. En realidad, el problema de la relación de fuerzas en un mercado se plantea en términos opuestos. En razón de su poder financiero, el conglomerado puede controlar un mercado en el cual tiene reducida participación, pues una de las armas que tiene en manos es exactamente la capacidad financiera para aumentar, si juzga necesario, su participación en el mercado. Actuando simultáneamente en múltiples mercados, el conglomerado dispone de múltiples opciones. Siempre podrá escoger el frente en que más le conviene tomar la iniciativa. Su visión global le permite actuar con mayor eficacia que las firmas en läs confrontaciones de mercado ya que éstas tienen recursos totalmente comprometidos en un solo sector. En relación a otro conglomerado, su capacidad estratégica es, evidentemente, mucho mayor que la de la firma especializada.

Los estudios empíricos de los procesos de conglomeración han puesto en evidencia que las finmas absorbidas; en general están lejos de ser empresas en declinación. En general los conglomerados en expansión buscan empresas en rápido crecimiento o que actúan en mercados cuyas potencialidades son reconocidas. También existe evidencia empírica de que las empresas que se lanzan por la vía de la conglomeración presentan la doble caractertstica de declinación en su tasa de crecimiento y de amplia disponibilidad de recursos liquidos. Al confrontarse con dificultades para continuar creciendo en su propio sector, determinada empresa busca la diversidad, y el camino de más fácil acceso a ésta es la adquisición de empresas con experiencia en 
otros sectores y posiciones ya afirmadas en otros mercados. Evidentemente, también existe la diversificación que resulta del aprovechamiento directo de subproductos y de rumbos inesperados de la propia investigación tecnológica. Empero, este tipo de diversificación es más una forma de crecimiento endógeno de la empresa, mientras que la conglomeración es principalmente un proceso de fusión con otras empresas.

'Señalamos que la conglomeración es un fenómeno del capitalismo postcíclico. También sería apropiado calificarlo de característica de la época del consumo dirigido. En la medida en que el mercado asume la forma de acción más o menos articulada de los productores para forzar al consumidor a diversificar permanentemente su consumo y mantenerlo a elevado nivel, el ciclo vital de cada producto tiende a reducirse. En consecuencia, el ciclo vital de cada línea de producción y, hasta cierto punto, de gran número de industrias, tiende a estrecharse. De ahí que tanto la obsolescencia rápida de los procesos tecnológicos como la sustitución acelerada de los artículos que llegan en manos del çonsumidor final, influyan en el sentido de favorecer las firmas que operan en un mayor número de frentes productivos. El ciclo vital de estas firmas se parece cada vez menos al de un producto o de una rama de industrias para asemejarse al del conjunto de la actividad manufacturera.

Las consideraciones que acabamos de hacer ponen en evidencia la necesidad de reconstrucción del marco teórico con que nos habituamos a trabajar, en lo que atañe a la organización de la producción en las economías capitalistas. La empresa típica de Marshall, entidad responsable de la organización de la producción, buscando su equilibrio en determinado mercado a través de la maximización de la tasa de ganancia, cuya estructura estaría dada por los precios relativos de los factores establecidos en los mercados y el dominio de una técnica, tiende a desaparecer como categoría analítica ${ }^{10}$.

El conglomerado ni se liga a un mercado, ni depende de una competencia técnica particular. Es, esencialmente, una amalgama de capacidad gerencial-administrativa y control de una masa crítica de recursos financieros. En una economía de precios administrados ${ }^{11}$, su problèma central es menos la maximización de la tasa de ganancias y más la búsqueda de aplicación remuneradora para el flujo de nuevos

\footnotetext{
${ }^{10} \mathrm{~A}$ ese respecto, es pionero el trabajo de François Perroux que en su teoría de la firma dominante ha dado énfasis a las potencialidades de la empresa como centro de poder y como instrumento transformador de las estructuras económicas, Véase L'Economie du XXème Silcle (Parts, 1965).

"2se estima que en los Estados Unidos cerca del 90 por ciento de los productos manufacturados tienen su precios fijados administrativamente, es decir, independientemente de una situación de mercado en el corto plazo.
} 
recursos bajo su control. Al contrario de la empresa marshalliana que era un instrumento para remunerar determinado monto de capital, el conglomerado moderno es principalmente un mecanismo para invertir en expansión un flujo de recursos creados por él mismo.

En una estructura económica de precios establecidos administrativamente $y^{\prime}$ en que las empresas se expanden principalmente en base a los recursos financieros que crean, la remuneración del capital pierde su carácter residual al nivel de la empresa. La aptitud para acumular recursos tiende a ser la característica principal de la empresa y el problema central de ésta viene a ser evitar que pase de ciertos límites su coeficiente de liquidez. En efecto, hay evidencia empirica de que las empresas que buscan el camino de la conglomeración son exactamente aquellas cuyo coeficiente de liquidez tiende a superar los límites considerados como razonables. La dirección de tales empresas pasa a dedicar parte creciente de su tiempo a problemas de orden financiero, lo que la induce a ampliar su horizonte de acción.

Así, el control de un flujo creciente de recursos líquidos y la posibilidad de condicionar el comportamiento del consumidor mediante medios de persuasión, son los factores determinantes del fenómeno de la conglomeración. El conglomerado es esencialmente un centro de decisiones de base gerencial-financiera: Para lograr invertir el flujo de recarsos líquidos que acumula, necesita diversificar su actuación, por el hecho mismo de que el consumo de la colectividad crece divercificándose. Al contribuir intensamente para diversificar y dinamizar el consumo, abre asimismo nuevas posibilidades de expansión. De ahí que las empresas especializadas y el mercado de capitales tiendan a ocupar un lugar cada vez menos relevante en la estructura económica, al propio tiempo que se amplían las avenidas al proceso de concentración.

La naturaleza y la amplitud de las decisiones que en sus manos tienen los grandes conglomerados, son hoy día objeto de creciente interés en los Estados Unidos. ¿Qué repercusiones tendrá en la sociedad estadounidense la modificación en la estructura del poder que está provocando la progresiva concentración del poder económico? Una vez abandonada la ficción de la spberanía del consumidor y de los automatismos de los mercados, colócase de forma directa el problema de encontrar una base de legitimidad para el poder de grupos que, bajo la forma de instituciones privadas, tienen en sus manos el control de un número creciente de decisiones de carácter público ${ }^{12}$.

12Adolph Berle indica que la dirección de una sociedad anónima es 'una oligarquía que se perpétúa a partir de sí misma y automáticamente'. Véase 'Economic Power and the Free Society' en The Corporalion Take-Over (New York, 1J64), p. 91. 
Conforme señala Kenneth Galbraith en libro reciente, los hombres en el futuro mirarán hacia atrás con aire burlón las razones que en el pasado llevaron la gente a referirse a la General Dynamics, la North American Aviation o la $A$. $T$. and $T$. como "empresas privadas"13.

\section{ProyecGiones en la AMérica latin'a}

El fenómeno de la conglomeración se ha presentado tanto bajo la forma de diferenciación funcional como de dispersión geagráfica, o aun bajo ambas formas combinadas. La empresa que actúa simultáneamente en distintos espacios económicos adquiere en cada uno de ellos, relativamente a los competidores locales, ventajas similares a las del conglomerado funcional que opera en mercados no directamente relacionados en un solo espacio económico. En los dos casos la fuerza principal del conglomerado radica en su gran poder financiero y en el hecho de que sus recursos están dispersos. Las ventajas de la dispersión geográfica son aún mayores que las de la diferenciación funcional, pues la experiencia obtenida en cierta linea de producción en un área puede ser utilizada en otras. Si se unen las dos formas de conglomeración - la funcional y la geográfica- se amplían las posibilidades de concentración del poder económico. En efecto, la gran unidad multifuncional y multigeográfica es la forma superior de organización de la economía capitalista.

La expansión del capital estadounidense hacia América latina se realiza, de manera casi exclusiva, bajo la forma de crecimiento de grandes conglomerados geográficos o mixtos: La estructura de esos conglomerados y el grado de concentración de poder económico que representan, no.han sido objeto de un examen sistemático hasta el presente. Sin embargo, estudios preliminares llevan a pensar que la expansión relativa de tales conglomerados es por lo menos tan grande en América latina como en los Estados Unidos. En efecto, el grado de concentración del sector de la economía latinoamericana formado por las empresas norteamericanas es mayor que el observado en Estados Unidos. Mientras en este país, en 1962, las 1:000 mayores empresas manufactureras controlaban-tres cuartas partes de las ventas totales, ya en 1950, en América latina, las 300 mayores firmas americanas contribuyen con el 90 por ciento del total de las inversiones realizadas por el grupo de firmas de propiedad.de ciudadanos estadounidenses ${ }^{14}$. Aunque se carezca hasta el presente de estudios sistemáticos sobre la materia, existe alguna evidencia empfrica que. lleva a admitir que

\footnotetext{
${ }^{12}$ J. K. Galbraith, The New Industrial Stale (Boston, 1967), passin.

- "Los datos relativos a América latina son de El Financiamiento Externo de América latina (Naciones Unidas, 1964), p. 238.
} 
empresas extranjeras -en su gran mayoria conglomerados norteamericanos- controlan del 50 al 75. por ciento de las industrias dinámicas, es decir, de las industrias que marchan a la cabeza en el proceso de desarrollo en América latina. Esas industrias crecen con tasa que es muy superior al promedio del incremento del sector manufacturero. Comparadas al conjunto de la economía, las industrias dinámicas crecen con una tasa de dos a cuatro veces mayor.

Los conglomerados geográficos y mixtos, que son parte del sistema económico de los Estados Unidos en un sentido amplio, constituyen hoy día elementos esenciales de los sistemas económicos nacionales latinoamericanos. Es dable, por lo tanto, admitir que son estas estructuras de nuevos tipos. ¿Hasta qué punto se puede continuar utilizando el concepto de sistema económico nacional considerando la realidad que se viene fraguando en América latina? Si se tiene en cuenta que los conglomerados geográficos definen su estrategia, uno con respecto a los demás y cada uno con respecto a competidores locales, con una perspectiva de conjunto y en función de un proyecto de erecimiento propio, no es fácil conciliar esa realidad con el concepto de sistema económico nacional que implica la idea de unificación de las decisiones en función de intereses específicos de una colectividad. Por otro lado, cabría preguntar: chasta qué punto ya no se comportan como estructura autónoma los conglomerados geográficos y mixtos que actúan en América latina? En la medida en que son competitivos o complementarios entre ellos mismos y que hacen frente a problemas comunes, los conglomerados, al definir su estrategia, son llevados a actuar como un sistema de decisiones, vale decir, como una estructura que se comporta con relativa autonomía vis-à-vis los sistemas económicos nacionales, en la medida en que estos últimos tienen una realidad propia.

La penetración de los conglomerados estadounidenses en las industrias manufactureras de América latina es fenómeno posterior a la gran depresión de la década de 1930. Es a partir de la segunda guerra mundial que esa penetración alcanza gran intensidad ${ }^{15}$, particularmente en aquellos paises que ya habían cumplido una primera fase de desarrollo industrial10. En esa forma, independientemente del grado de desarrollo industrial que hubiera alcanzado el país, hubo por todas partes una interrupción en el proceso de formación de una clase

\footnotetext{
${ }^{15 E n} 1929$ las inversiones directas en manufacturas latinoamericanas representaban el 6,3 por ciento del total de esas inversiones. En el periodo 1951-62 las manulacturas absorberán el 31 por ciento del total. Véase El Financiamiento Externo de América latina, cit., cuadros 15 y 179 .

${ }^{10 E} 1929$ la participación de la producción industrial en el producto interno bruto ya cra, en la Argentina, de 22,8 por ciento; en México de 14,2 y en Brasil de 11,7.
}

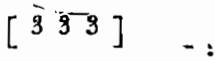


de empresarios industriales autónomos. De las fuertes posiciones financieras que ocupan, los conglomerados extendieron progresivamente su control sobre los sectores más dinámicos de las manufacturas. Los hombres más capaces surgidos de las industrias locales fueron absorbidos en la nueva clase gerencial, asimilando rảpidamente los patrones profesionales que les permiten ascender en la jerarquía de la nueva estructura de poder económico. La acción empresarial nacional independiente queda así restringida a sectores secundarios o a formas de acción pionera que, a la larga, se transforma en trabajo de limpieza del terreno para la expansión futura de los conglomerados estadounidenses.

Si se admite que en las estructuras económicas latinoamericanas está entretejida una estructura de conglomerados geográficos y mixtos, cuyo centro hegemónico se encuentra fuera del área, es dable suponer que un proceso de integración en dos dimensiones se realiza actualmente: integración dentro de la región y de ésta con los Estados Unidos. No estamos en condición de captar la verdadera naturaleza de ese complejo proceso de integración, que es mucho más un sistema de decisiones creado por la concentración del poder económico, que un espacio económico amplificado para obtención de una utilización más racional de recursos escasos. Ese sistema de decisiones opera como una superestructura regional, cuya función principal parecería ser abrir camino a la concentración del poder económico en rápida realización en el sistema económico hegemónico.

A la luz de estas consideraciones, parece ocioso inquirir si existe o. no en los países latinoamericanos una clase empresarial autónoma (una burguesía industrial nacional, en la expresión mảs popular) capaz de encabezar el proceso de desarrollo. El estudio de las estructuras actuales pone en evidencia que una tal clase, alli donde ella haya podido existir, ya no encontrarla condiciones para realizax el papel que históricamente se le atribuye. Los agentes económicos que operan en el marco de una estructura, pueden o no participar de forma relevante en el proceso de modificación de esa estructura. Todo indica que, en la medida en que se desarrolló la superestructura de decisiones formada por los poderosos conglomerados que trabajan simultáneamente en los distintos paises, las decisiones de largo plazo con impacto en las estructuras se fueron desplazando del grupo de empresarios autónomos hacia la nueva clase de gerentes. Al mismo tiempo, tales decisiones dejaron de reflejar un proyecto nacional de desarrollo para traducir las tendencias del complejo proceso de integración a que se ha hecho referencia más arriba. 
La idea corriente de que los empresarios nacionales autónomos intentarían defender sus intereses de la intrusión de los grandes conglomerados controlados del exterior se funda en simple razonamiento analógico con el conflicto tradicional entre el proteccionismo de esos empresarios y el libre-cambismo de los intereses ligados a las importaciones de manufacturas. En este último caso, el empresario local se identificaba fácilmente con los sentimientos nacionalistas, y la defensa de la empresa nacional se traducía en defensa de las fuentes de empleo. La penetración de los conglomerados se tradujo en la implantación de industrias modernas, en baja de los precios relativos de los productos de prestigio que consume la clase media, etc. Se explica aś que los empresarios autónomos -cuando habían llegado a alcanzar una percepción mayor del problema- hayan quedado incapacitados para traducir sus intereses en términos de aspiraciones nacionales. Además, la penetración se hizo en general en periodos de intenso desarrollo del sector industrial, lo que permitía a los empresarios locales hacer 'buenos negocios' sin dárse cuenta de los cambios estructurales que estaban en marcha ${ }^{17}$.

La eliminación o anulación de la clase de empresarios nacionales autónomos excluye necesariamente la posibilidad de un desarrollo autogenerado, en la línea del capitalismo clásico. En el correr del último cuarto de siglo, siempre que el desarrollo latinoamericano tuvo que apoyarse en la industrialización, reflejó la estrategia de la superestructura de conglomerados geográficos (es decir, integración con el sistema económico hegemónico) o la acción del Estado nacional. En la medida en que se implantan los conglomerados, las empresas locales pasan a actuar de forma refleja, cumpliendo tareas auxiliares o limitándose a los sectores estancados. Las únicas empresas que disponen de recursos financieros para seguir creciendo en posiciones estratégicas, son aquellas que apoya directamente el Estado. Empresas como Pemex, Gap, Volta Redonda, ENAP, Vale do Rio Doce y otras, no hubieran existido y sobrevivido sin el apoyo decidido de los respectivos Estados nacionales. Es dable esperar, por lo tanto, que la interacción de los dos sistemas de decisiones - la superestructura de conglomerados y los Estados nacionales- continúe a definir, en los próximos años, las posibilidades y las formas del desarrollo económico en América latina. Solamente en aquellos casos en que se presente la posibilidad de ampliar y racionalizar la acción del Estado como cen-

17EI carácter familiar que neccsariamente tenían las emplesas latinoamericanas, en razón de su creación reciente, facilitó la penetración de los congloncrados. El recmplazo de la prineza gencración de empresarios por la segunda, en el cuadro familiar, constituye el punto crítico de la evolución de toda empresa. Es corriente que la segunda generación tenga vocación para rentista, lo que facilita la transferencia del control de la dirección. 
tro de decisiones autónomo -es decir, independiente del otro sistema de decisiones formado por los conglomerados- el desarrollo existirá como una opción al alcance de la colectividad nacional. El debilitamiento del Estado como centro autónomo de decisiones no implica necesariamente, en todos los casos, desarrollo más lento o estancamiento. Pero el desarrollo ya no será una opción. Será consecuencia posible de un proceso histórico extraño a la comunidad, al igual que las transformaciones de una economía dependiente tradicional.

Cabe admitir que, en la medida en que se tome plena conciencia de ese problema, una nueva temática ligada al desarrollo venga a ser planteada en la América latina. Así, el alcance, la naturaleza y los riesgos de la llamada polftica de integración regional, podrán ser considerados bajo nueva luz. Lo mismo se puede decir con respecto a la controversia en torno al papel del Estado en el desarrollo, y a la búsqueda de bases para el sistema de poder que aumenten su independencia vis-à-vis las fuerzas ligadas a la superestructura de conglomerados. Por último, como la eliminación del desarrollo en base a un proyecto nacional no sería compatible con la preservación de la identidad cultural como posibilidad histórica, se puede admitir como probable que, polftica de desarrollo -allí en donde ésta sea una ex. presión de aspiraciones de la colectividad- y lucha por la preservación de la personalidad nacional, tiendan a confundirse en la América latina. 